\title{
Application of 3D printing and its various technologies in dentistry
}

\author{
Rajeh M. Al-Sharif ${ }^{1 *}$, Khaled A. Althaqafi ${ }^{2}$, Hend S. Alkathiry ${ }^{3}$, Abdulrahman A. Alzeer ${ }^{4}$, \\ Raiya M. Shareef ${ }^{5}$, Samia M. Omran ${ }^{5}$, Zahra M. Alabduljabbar6 ${ }^{6}$, Asmaa A. Alnuman ${ }^{7}$, \\ Abdullah M. Alshahrani ${ }^{8}$, Abeer M. Alharbi ${ }^{7}$, Saad H. Alqahtani ${ }^{9}$, Haya T. Alsharif ${ }^{10}$
}

\author{
${ }^{1}$ North Jeddah Specialist Dental Center, King Abdullah Medical Complex, Jeddah, Saudi Arabia \\ ${ }^{2}$ College of Dentistry, Umm Al-Qura University, Makkah, Saudi Arabia \\ ${ }^{3}$ General Dentist, Dr. Sulaiman AlHabib Hospital, Riyadh, Saudi Arabia \\ ${ }^{4}$ General Dentist, Ministry of Health, Riyadh, Saudi Arabia \\ ${ }^{5}$ Primary Healthcare, Ministry of Health, Makkah, Saudi Arabia \\ ${ }^{6}$ General Dentist, Prince Sultan Hospital, Uraira, Saudi Arabia \\ ${ }^{7}$ College of Dentistry, Hail University, Hail, Saudi Arabia \\ ${ }^{8}$ General Dentist, Al-Nomais Medical Group, Abha, Saudi Arabia \\ ${ }^{9}$ College of Dentistry, King Khalid University, Abha, Saudi Arabia \\ ${ }^{10}$ General Dentist, Ideal Clinics, Jeddah, Saudi Arabia
}

Received: 31 December 2021

Accepted: 05 January 2022

*Correspondence:

Dr. Rajeh M. Al-Sharif,

E-mail: dr.rmsh@hotmail.com

Copyright: (C) the author(s), publisher and licensee Medip Academy. This is an open-access article distributed under the terms of the Creative Commons Attribution Non-Commercial License, which permits unrestricted non-commercial use, distribution, and reproduction in any medium, provided the original work is properly cited.

\begin{abstract}
Many applications for these technologies have been reported in multiple fields, including dentistry, within the last three decades. It can be used in periodontology, endodontics, orthodontics, oral implantology, maxillofacial and oral surgery, and prosthodontics. In the present literature review, we have discussed the different clinical applications of various 3D printing technologies in dentistry. Evidence indicates that 3D printing approaches are usually associated with favorable outcomes based on the continuous development and production of novel approaches, enabling clinicians to develop complex equipment in different clinical and surgical aspects. Developing work models to facilitate diagnostic and surgical settings is the commonest application of these modalities in dentistry. Besides, they can also be used to manufacture various implantable devices. Accordingly, they significantly help enhance the treatment process, reducing costs and less invasive procedures with favorable outcomes. Finally, 3D printing technologies can design complex devices in a facilitated and more accurate way than conventional methods. Therefore, 3D printing should be encouraged in clinical settings for its various advantages over conventional maneuvers.
\end{abstract}

Keywords: 3D printing, Dentistry, Clinical application, Efficacy

\section{INTRODUCTION}

Evidence shows that the $1^{\text {st }} 3 \mathrm{D}$ printing technology was introduced in 1986. After which, many advances were reported in multiple fields with multiple clinical applications. ${ }^{1,2}$ In this context, Huller created and developed a $3 \mathrm{D}$ printing system was created and developed in 1986 Huller, which also printed stereolithography (SLA). Moreover, fused deposition modeling (FDM) was first introduced by Scott Crump in $1990 .^{3}$ Since these advances, many technological breakthroughs have been reported for 3D printing technologies.

3D printing can be used to develop personalized 3D subjects according to computer-aided design (CAD) digital approaches via particular automatic processes and standardized materials. ${ }^{4-6}$ Many applications for these technologies have been reported within the last three 
decades in multiple fields, like manufacturing, engineering, design, and industry. In addition, it has also been used in various medical fields, like craniomaxillofacial surgery, plastic surgery, neurosurgery, cardiology, traumatology, patient-physician communication, customizing surgical devices, and digital imaging in surgical planning. ${ }^{7}$ Furthermore, many applications have also been reported in the field of dentistry, including periodontology, endodontics, orthodontics, oral implantology, maxillofacial and oral surgery, and prosthodontics. ${ }^{8,9}$ In the present literature review, we will discuss the various clinical applications of $3 \mathrm{D}$ printing technologies in dentistry based on the results and outcomes of previous relevant studies.

\section{METHODS}

This literature review is based on an extensive literature search in Medline, Cochrane, and EMBASE databases on which was performed 03 December 2021 using the medical subject headings (MeSH) or a combination of all possible related terms, according to the database. To avoid missing poetential studies, a further manual search for papers was done through Google Scholar, while the reference lists of the initially included papers. Studies discussing application of 3D printing and its various technologies in dentistry were screened for useful information, with no limitations posed on date, language, age of participants, or publication type.

\section{DISCUSSION}

Among the various investigations in the literature, it has been shown that different clinical applications have been proposed for $3 \mathrm{D}$ techniques. In the present section, we will discuss these applications based on data from relevant studies in the literature. However, the commonest applications for 3D printing technologies appear to be used for surgical treatment and diagnostic purposes. Besides, it has been shown that these technologies can be furtherly used in different fields, including oral implantology, maxillofacial surgery, prosthodontics, and others.

For instance, it has been demonstrated that 3D technologies can be effectively used in prosthodontics to scan, prepare, and print teeth immediately in one session during the clinical setting. Accordingly, these technologies can effectively save time and cost. ${ }^{10}$ In addition, clinicians can effectively prepare optimal teeth models by using extraoral or intraoral scanners. CAD software can be used to achieve favorable restorations and treatment outcomes. It has been shown that these applications can be used to print various dental restorations. ${ }^{11}$ In this context, evidence indicates that resin-based 3D technologies (including DLP and SLA) can be effectively used to fabricate crown and bridge dentures. ${ }^{12,13}$ A previous study reported that the main advantage of using these technologies is minimal loss of materials and the number of materials used compared with milling. ${ }^{14}$ These modalities can also obtain favorable detail reproducibility when aiming to print various materials simultaneously. A previous investigation by Wang et al. ${ }^{15}$ evaluated the authentic actuality of milled crowns and 3D printing crowns and found that the external trueness of both modalities was remarkably similar. A good fit is recommended when planning for the treatment strategy to maintain the surrounding tissues' health, durability, and mechanical stability. ${ }^{10}$ If the materials were not adequately fit, various adverse events could develop in this context. These include the development of periodontal diseases, dental caries, tooth sensitivity, lack of esthetics, discoloration, microleakage of adhesive, and dental plaque accumulation over the edges. ${ }^{16}$

Many studies demonstrated that the application of 3Dbased temporary crowns significantly achieved enhanced internal fit abilities and excellent edges. These effects were more significant than those obtained when applying temporary crowns manufactured by traditional milling approaches and CAD/computer-aided manufacturing. ${ }^{17}$ This has been further indicated in a previous investigation by Pomba et al which compared the differences between 3D printing, molding, and milling methods regarding internal fit and edges of fabricated temporary crowns. ${ }^{18}$ The most favorable events were associated with the molding method followed by $3 \mathrm{D}$ printing, which might be attributed to volume shrinkage during polymerization. Other studies even reported that the differences were even greater when it was aggregated within a single piece. ${ }^{18}$ Another study by Chaturvedi et al further reported that 3D printing was significantly associated with enhanced coordination of interior, edge, and proximal ends of the temporarily fabricated crowns. ${ }^{11}$ On the other hand, another study by Alharbi et al concluded that the internal clearance values and edges were remarkably lower for 3D printing than milled restorations. ${ }^{5}$ However, this has been attributed to the errors associated with milling tool tolerances. ${ }^{19,20}$

Evidence indicates that 3D printing techniques can also fabricate complete resin-based dentures with reduced need for tooling fixtures, cutting tools, or molds. It has been furtherly shown that other modalities rather than 3D printing technologies (like traditional curing with heat, subtraction technology, and self-curing modalities) might additionally be applied in fabricating complete denture bases. ${ }^{21}$ Studies also showed that combining compression molding technology and polymethylmethacrylate is common. However, these studies showed that these modalities' linear shrinkage and volume are higher than 3D printing techniques. ${ }^{22}$ On the other hand, 3D printing technologies are usually associated with a reduced frequency of errors secondary to reduced working stages and faster production of dentures. ${ }^{23}$ However, evidence shows that applying these modalities in this field is still under investigation. It should be noted that adequate tissue adaptation is vital for the stability of removable dentures, performance retention, and masticatory. ${ }^{24}$ In this context, a previous study by Tasaka et al concluded that photopolymerization spray was significantly able to fabricate complete denture bases that are more accurate 
than the ones obtained by conventional thermal polymerization..$^{25}$

Using 3D printing was also efficiently reported in obtaining removable partial denture frameworks. Various investigations in the literature also indicate that the modality is usually associated with more favorable outcomes than conventional approaches. For instance, it has been shown that these conventional modalities are usually associated with poor casting fit more significantly than 3D printing approaches. ${ }^{26}$ Besides, studies also reported that the associated clinical complications are mainly attributed to residual ridge resorption and pressureinduced mucosal lesions. In this context, evidence indicates that $3 \mathrm{D}$ printing techniques significantly reduce the risk of long-term bone resorption and provide an enhanced and uniform contact pressure. A previous investigation reported that SLM obtained clinicallyacceptable removable partial dentures. ${ }^{27}$ Another study by Tregerman et al showed that enhanced mechanical and organization characteristics were more significant with SLM Co-Cr alloys than milled or cast removable partial dentures. $^{28}$

There is also evidence of the efficacy of applying 3D printing technologies and CAD/CAM technologies in conducting efficacious maxillofacial and oral surgeries. Many advantages have been reported for using these modalities in maxillofacial surgeries. For instance, it has been demonstrated that they can significantly enhance the functional impacts and symmetry of maxillofacial and oral surgeries and the associated techniques. ${ }^{29}$ On the other hand, some studies reported that milling approaches for manufacturing the occlusal splints might be associated with a remarkable waste in materials and time-consuming. Besides, the process can be associated with filling errors that can also reduce the efficiency of these modalities and limit their therapeutic efficacies. ${ }^{30}$ On the other hand, when using 3D printing technologies, evidence shows that clinicians can manufacture several splints simultaneously. Accordingly, it has been reported that this is usually associated with favorable therapeutic outcomes, in addition to being time and cost-saving. ${ }^{31}$ Unfortunately, many previous investigations have reported that $3 \mathrm{D}$ printing materials do not usually have antiaging and antistress features similar to those with milling resin and traditional materials. ${ }^{32}$ Accordingly, there have many concerns about the long-term efficacy of these modalities. A previous study by Lutz et al compared the efficacy of traditionally made, milled, and 3D printed occlusal splints. ${ }^{33}$ The authors reported that flexure resistance and wear were significantly lower in 3D printed appliances than other approaches. Besides, it has been shown that these materials can be effectively used with a remarkable clinical efficacy for one month. ${ }^{33}$ However, it should be noted that the accuracy and milling and 3D printed splint are similar. ${ }^{34}$ It should be noted that the differences in materials and technologies can significantly impact the occlusal splints' performance. Evidence also indicates the efficacy of 3D printing technologies in surgical implants.
Studies showed that reconstruction and manufacturing of customized porous implants, as customized Ti mesh, can be effectively done by direct beam melting and laser sintering. ${ }^{35}$ A previous study further demonstrated that SLS technologies successfully manufactured bone conductive and completely biodegradable nanocomposite scaffold mechanical characteristics and adjustable porosity. ${ }^{36}$ In a previous study by Farré-Guasch et al, the authors reported that laser sintering technologies could successfully induce mandible formation. ${ }^{36} \mathrm{~A}$ new customized bone was also implanted for a patient with facial deformities by an inkjet printer using an $\alpha$-tricalcium phosphate powder.

Different studies in the literature explored the efficacy of 3D printing modalities in dental implantology and their abilities to manufacture accurate and digital models that are more efficacious than the traditional ones. Accordingly, many benefits can be obtained from applying these modalities. For instance, the efficiency of dentists can be significantly enhanced, the technical risk and technical difficulty can also be remarkably reduced, and the medical treatment process can furtherly be simplified and optimized. In addition, 3D printing techniques can be effectively used in the laboratory to manufacture static surgery guides or SLA guides, usually done by inducing perforations in jaw models. Moreover, previous studies indicate that the static surgical guide does not move during operations, which differs from the dynamic surgical guide.

Long-term stabilization of soft and hard tissues around dentures, achieving good esthetic effects, and simplifying the process of denture restoration can be achieved by the placement of an optimal dental implant. ${ }^{37}$ On the other hand, studies show that the prosthesis's reduced long-term predictability and success rates can result from improper dental implant placement. ${ }^{38}$ Accordingly, it is important to optimize the accuracy of surgical guides. A previous investigation also demonstrated that static-guided surgeries had less correlation error than real-time navigation. ${ }^{39}$ On the other hand, another study by Jung et al reported no significant difference between the two approaches. ${ }^{40}$ Another in vitro study by Tahmaseb et al compared the accuracy of conventional surgical guides with SLA guides. ${ }^{41}$ It has been shown that the average deviation at the vertex and entrance was $2.1 \mathrm{~mm}$ and 1.5 $\mathrm{mm}$, and $1.0 \mathrm{~mm}$ and $0.9 \mathrm{~mm}$ for traditional surgical guides and SLA guides, respectively. ${ }^{42}$ Accordingly, it has been concluded that SLA guides had better accuracy than traditional surgical guides. Recent advances in 3D printing were also reported in obtaining successful custom trays. Many studies have reported that these modalities have simpler personalization and programming and reduced processing durations. It has been furtherly reported that $3 \mathrm{D}$ printing modalities can adequately complete all the production processes. ${ }^{43}$ Therefore, they can be used for completely edentulous patients to make the workflow of direct implant-supported dentures simpler. A previous study compared the efficacy and accuracy of 3D printing modalities and traditional hand-made custom trays and 
reported that $3 \mathrm{D}$ printing technologies had better accuracy. In this context, many advantages have been proposed for 3D printed custom trays, including greater clinical satisfaction, high precision, and personalization than traditional custom trays. ${ }^{44-47}$ Accordingly, these advantages allow for 3D printing technologies to be widely used in this field with favorable outcomes. Future implications should also encourage the clinical application of these modalities. ${ }^{48-50}$

\section{CONCLUSION}

Evidence indicates that 3D printing approaches are usually associated with favorable outcomes based on the continuous development and production of novel approaches, enabling clinicians to develop complex equipment in different clinical and surgical aspects. Developing work models to facilitate diagnostic and surgical settings is the commonest application of these modalities in dentistry. Besides, they can also be used to manufacture various implantable devices. Accordingly, they significantly help enhance the treatment process, reducing costs and less invasive procedures with favorable outcomes. Finally, 3D printing technologies can design complex devices in a facilitated and more accurate way than conventional methods. Therefore, using 3D printing should be encouraged in clinical settings for its various advantages over conventional maneuvers.

\section{Funding: No funding sources \\ Conflict of interest: None declared \\ Ethical approval: Not required}

\section{REFERENCES}

1. Barazanchi A, Li KC, Al-Amleh B, Lyons K, Waddell JN. Additive technology: update on current materials and applications in dentistry. J Prosthodont. 2017;26(2):156-63.

2. Mai H-N, Lee K-B, Lee D-H. Fit of interim crowns fabricated using photopolymer-jetting $3 \mathrm{D}$ printing. $\mathrm{J}$ Prosthetic Dentistry. 2017;118(2):208-15.

3. Gross BC, Erkal JL, Lockwood SY, Chen C, Spence DM. Evaluation of $3 \mathrm{D}$ printing and its potential impact on biotechnology and the chemical sciences. In: ACS Publications. 2014.

4. Vukicevic M, Mosadegh B, Min JK, Little SH. Cardiac 3D printing and its future directions. JACC Cardiovasc Imag. 2017;10(2):171-84.

5. Alharbi N, Alharbi S, Cuijpers VM, Osman RB, Wismeijer D. Three-dimensional evaluation of marginal and internal fit of 3D-printed interim restorations fabricated on different finish line designs. J Prosthodont Res. 2018;62(2):218-26.

6. Lin L, Fang Y, Liao Y, Chen G, Gao C, Zhu P. 3D printing and digital processing techniques in dentistry: A review of literature. Adv Eng Mat. 2019;21(6):1801013.
7. Lin $\mathrm{H}-\mathrm{H}$, Lonic $\mathrm{D}$, Lo L-J. $3 \mathrm{D}$ printing in orthognathic surgery- A literature review. J Formosan Med Assoc. 2018;117(7):547-58.

8. Prechtel A, Reymus M, Edelhoff D, Hickel R, Stawarczyk B. Comparison of various 3D printed and milled PAEK materials: Effect of printing direction and artificial aging on Martens parameters. Dent Mat. 2020;36(2):197-209.

9. Oberoi G, Nitsch S, Edelmayer M, Janjić K, Müller AS, Agis H. 3D Printing - encompassing the facets of dentistry. Front Bioeng Biotech. 2018;6:172.

10. Sampaio CS, Niemann KD, Schweitzer DD, Hirata R, Atria PJ. Microcomputed tomography evaluation of cement film thickness of veneers and crowns made with conventional and 3D printed provisional materials. J Esthetic Restorative Dentistry. 2021;33(3):487-495.

11. Chaturvedi S, Alqahtani NM, Addas MK, Alfarsi MA. Marginal and internal fit of provisional crowns fabricated using 3D printing technology. Technol Health Care. 2020;28(6):635-42.

12. Munoz S, Dickinson DP. Comparison of margin discrepancy of complete gold crowns fabricated using printed, milled, and conventional hand-waxed patterns. J Prosthetic Dentistry. 2017;118(1):89-94.

13. Park J-Y, Lee J-J, Bae S-Y, Kim J-H, Kim W-C. In vitro assessment of the marginal and internal fits of interim implant restorations fabricated with different methods. J Prosthetic Dentistry. 2016;116(4):536-42.

14. Van Noort R. The future of dental devices is digital. Dent Materials. 2012;28(1):3-12.

15. Wang W, Yu H, Liu Y, Jiang X, Gao B. Trueness analysis of zirconia crowns fabricated with 3dimensional printing. J Prosthetic Dentistry. 2019;121(2):285-91.

16. Burns DR, Beck DA, Nelson SK. A review of selected dental literature on contemporary provisional fixed prosthodontic treatment: report of the Committee on Research in Fixed Prosthodontics of the Academy of Fixed Prosthodontics. J Prosthetic Dentistry. 2003;90(5):474-97.

17. Lee W-S, Lee D-H, Lee K-B. Evaluation of internal fit of interim crown fabricated with CAD/CAM milling and 3D printing system. J Adv Prosthodont. 2017;9(4):265-70.

18. Pompa G, Di Carlo S, De Angelis F, Cristalli MP, Annibali S. Comparison of conventional methods and laser-assisted rapid prototyping for manufacturing fixed dental prostheses: an in vitro study. BioMed Res Int. 2015.

19. Beuer F, Schweiger J, Edelhoff D. Digital dentistry: an overview of recent developments for CAD/CAM generated restorations. $\mathrm{Br}$ Dent J. 2008;204(9):505-11.

20. Ortorp A, Jonson D, Mouhsen A. The t of cobaltchromium three unit xed dental prostheses fabricated with 4 different techniques: a comparative in vitro study. Dent Mat J. 2011;27:356-63. 
21. De Bruyne M, De Moor R, Raes F. Necrosis of the gingiva caused by calcium hydroxide: a case report. Int Endodont J. 2000;33(1):67-71.

22. Parvizi A, Lindquist T, Schneider R, Williamson D, Boyer D, Dawson DV. Comparison of the dimensional accuracy of injection-molded denture base materials to that of conventional pressure-pack acrylic resin. J Prosthodont Implant Esthetic Reconstruct Dentistry. 2004;13(2):83-9.

23. Khng KYK, Ettinger RL, Armstrong SR, Lindquist T, Gratton DG, Qian F. In vitro evaluation of the marginal integrity of CAD/CAM interim crowns. J Prosthetic Dentistry. 2016;115(5):617-23.

24. Yoon S-N, Oh KC, Lee SJ, Han J-S, Yoon H-I. Tissue surface adaptation of CAD-CAM maxillary and mandibular complete denture bases manufactured by digital light processing: A clinical study. J Prosthetic Dentistry. 2020;124(6):682-9.

25. Tasaka A, Matsunaga S, Odaka K. Accuracy and retention of denture base fabricated by heat curing and additive manufacturing. $\mathrm{J}$ Prosthodont Res. 2019;63(1):85-9.

26. Bilgin MS, Baytaroğlu EN, Erdem A, Dilber E. A review of computer-aided design/computer-aided manufacture techniques for removable denture fabrication. Eur J Dentistry. 2016;10(02):286-91.

27. Chen J, Ahmad R, Suenaga H. Shape optimization for additive manufacturing of removable partial dentures-a new paradigm for prosthetic CAD/CAM. PloS One. 2015;10(7):e0132552.

28. Tregerman I, Renne W, Kelly A, Wilson D. Evaluation of removable partial denture frameworks fabricated using 3 different techniques. J Prosthetic Dentistry. 2019;122(4):390-5.

29. Louvrier A, Marty P, Barrabé A, Euvrard E, Chatelain B, Weber E, Meyer C. How useful is 3D printing in maxillofacial surgery? J Stomatol Oral Maxillofac Surg. 2017;118(4):206-12.

30. Salmi M, Paloheimo K-S, Tuomi J, Ingman T, Mäkitie A. A digital process for additive manufacturing of occlusal splints: a clinical pilot study. J Royal Society Interface. 2013;10(84):20130203.

31. Berli C, Thieringer FM, Sharma N, et al. Comparing the mechanical properties of pressed, milled, and 3Dprinted resins for occlusal devices. J Prosthetic Dentistry. 2020;124(6):780-6.

32. Reymus M, Stawarczyk B. In vitro study on the influence of postpolymerization and aging on the Martens parameters of 3D-printed occlusal devices. J Prosthetic Dentistry. 2021;125(5):817-23.

33. Lutz A-M, Hampe R, Roos M, Lümkemann N, Eichberger M, Stawarczyk B. Fracture resistance and 2-body wear of 3-dimensional-printed occlusal devices. J Prosthetic Dentistry. 2019;121(1):166-72.

34. Marcel R, Reinhard H, Andreas K. Accuracy of CAD/CAM-fabricated bite splints: milling vs 3D printing. Clin Oral Investig. 2020;24(12):4607-15.

35. Ciocca L, Fantini M, De Crescenzio F, Corinaldesi G, Scotti R. Direct metal laser sintering (DMLS) of a customized titanium mesh for prosthetically guided bone regeneration of atrophic maxillary arches. Med Biol Eng Comp. 2011;49(11):1347-52.

36. Farré-Guasch E, Wolff J, Helder MN, Schulten EA, Forouzanfar T, Klein-Nulend J. Application of Additive Manufacturing in Oral and Maxillofacial Surgery. J Oral Maxillofac Surg. 2015;73(12):2408-18.

37. Chen X, Yuan J, Wang C, Huang Y, Kang L. Modular preoperative planning software for computer-aided oral implantology and the application of a novel stereolithographic template: a pilot study. Clin Implant Dentistry Relat Res. 2010;12(3):181-93.

38. Lal K, White GS, Morea DN, Wright RF. Use of stereolithographic templates for surgical and prosthodontic implant planning and placement. Part I. The concept. J Prosthodont. 2006;15(1):51-8.

39. Hermann JS, Schoolfield JD, Schenk RK, Buser D, Cochran DL. Influence of the size of the microgap on crestal bone changes around titanium implants. A histometric evaluation of unloaded non-submerged implants in the canine mandible. J Periodontol. 2001;72(10):1372-83.

40. Tahmaseb A, Wismeijer D, Coucke W, Derksen W. Computer technology applications in surgical implant dentistry: a systematic review. Int J Oral Maxillofac Implants. 2014;29:25-42.

41. Vercruyssen M, Hultin M, Van Assche N, Svensson K, Naert I, Quirynen M. Guided surgery: accuracy and efficacy. Periodontology 2000. 2014;66(1):228-46.

42. Al-Harbi SA, Sun AY. Implant placement accuracy when using stereolithographic template as a surgical guide: preliminary results. Implant Dentistry. 2009;18(1):46-56.

43. Skjerven H, Riis UH, Herlofsson BB, Ellingsen JE. In Vivo Accuracy of Implant Placement Using a Full Digital Planning Modality and Stereolithographic Guides. Int $\mathrm{J}$ Oral Maxillofac Implants. 2019;34(1):124-32.

44. Son PT, Reda A, Viet DC, Quynh NXT, Hung DT, Tung TH, Huy NT. Exchange transfusion in the management of critical pertussis in young infants: a case series. Vox Sang. 2021;116(9):976-82.

45. Nguyen TM, Huan VT, Reda A, Morsy S, Nam Giang $\mathrm{HT}$, Tri VD, $\mathrm{t}$ al. Clinical features and outcomes of neonatal dengue at the Children's Hospital 1, Ho Chi Minh, Vietnam. J Clin Virol. 2021;138:104758.

46. Pham TS, Reda A, Ngan Nguyen TT, Ng SJ, Huan VT, Viet DC, Huy NT. Blood exchange transfusion in viral hepatitis in a small infant: a case report. Transfus Apher Sci. 2020;59(6):102907.

47. Thieu H, Bach Dat B, Nam NH, Reda A, Duc NT, Alshareef A, et al. Antibiotic resistance of Helicobacter pylori infection in a children's hospital in Vietnam: prevalence and associated factors. Minerva Med. 2020;111(5):498-501.

48. Tian Y, Chen C, Xu X. A Review of 3D Printing in Dentistry: Technologies, Affecting Factors, and Applications. Scanning. 2021;9950131. 
49. Hosseini S, Halvaei M, Ebrahimi A, Shamekhi MA, Baghaban Eslaminejad M. 3D Printing in Dentistry. In: Tayebi L, ed. App Biomed Eng Dentistry. Cham: Springer International Publishing. 2020;195-221.

50. Dawood A, Marti BM, Sauret-Jackson V, Darwood A. 3D printing in dentistry. $\mathrm{Br}$ Dent $\mathrm{J}$. 2015;219(11):521-9.
Cite this article as: Al-Sharif RM, Althaqafi KA, Alkathiry HS, Alzeer AA, Shareef RM, Omran SM, et al. Application of 3D printing and its various technologies in dentistry. Int J Community Med Public Health 2022;9:937-42. 